\title{
ON THE POSSIBILITY OF REDUCING THE SAMPLE SIZE FOR MICROBIOLOGICAL EXAMINATION OF SOME PHARMACEUTICAL SUBSTANCES
}

\author{
M. V. Roshchina, ${ }^{1}$ O. V. Gunar, ${ }^{1, *}$ and I. A. Builova ${ }^{1}$
}

Translated from Khimiko-Farmatsevticheskii Zhurnal, Vol. 55, No. 9, pp. 61 - 64, September, 2021.

Original article submitted May 27, 2021.

The possibility of reducing the sample size for microbiological examination of some pharmaceutical substances is considered. Trypticase-soy agar was evaluated for the ability to detect yeast and mold fungi and to isolate the minimal amount of certain types of microorganisms. The minimal sample size for microbiological examination was $0.2 \mathrm{~g}(\mathrm{~mL})$ for synthetic substances intended for the production of non-sterile drugs and $1.2 \mathrm{~g}$ $(\mathrm{mL})$, for sterile drugs. Based on the experimental results, a scheme has been developed for microbiological examination of particular pharmaceutical substances using a reduced sample size

Keywords: pharmaceutical substances, validation, microbiological examination of non-sterile products, reduction of sample size for analysis.

One of the possible reasons for incidents of bacterial and fungal contamination of drugs in the middle of the $20^{\text {th }}$ century was the use of raw materials and pharmaceutical substances (PSs) during drug manufacturing that did not comply with the corresponding regulations.

Microbiological purity is the most important parameters characterizing drug safety. PSs for analysis of the Microbiological Purity parameter require a sample mass (volume) from 10 to $45 \mathrm{~g}(\mathrm{~mL})$, depending on the source and production method [1].

However, the analysis of several active pharmaceutical ingredients according to standard conditions is unacceptable because of the small volume of manufactured PS, their high intrinsic value (e.g., monoclonal antibodies), drugs intended for manufacturing that are included in list of orphan drugs and most expensive disease classes [2, 3]. Drugs intended for prevention and treatment of disease received special attention during the COVID-19 epidemic [4]. The production of substances to manufacture such drugs can be expensive and time-consuming [5] although many people experience a vital

\footnotetext{
${ }^{1}$ Scientific Center for Expert Evaluation of Medicinal Products, Ministry of Health of the Russian Federation, 8/2 Petrovskii Blvd., Moscow, 127051 Russia.

* e-mail: gunar@expmed.ru
}

need for these drugs that can ameliorate and/or avoid complications associated with a novel coronavirus infection.

Therefore, the possibility of reducing the sample mass (volume) for analysis remains a reasonable issue. The section Drug Sample Selection of the SP, XIIIth and XIVth Eds. includes GPM Microbiological Purity that states, "in some cases, the sample can be reduced to $2-3 \mathrm{~g}(\mathrm{~mL})$. The reduction of sample size with an indication of the method should be justified and approved in the regulations as customary." [1]. Builova performed a series of scientific studies addressing the possibility of reducing the sample size to $0.4 \mathrm{~g}$ to determine the total number of aerobic microorganisms and the total number of yeast and mold fungi [6]. The results were disclosed as patents No. 2,596,399 of Sept. 10, 2016, "Method of determining microbiological purity of pharmaceutical substance, produced biotechnologically" [7, 8] and No. 2,596,395 of Sept 10, 2016, "Method of determining microbiological purity of drugs of target therapy" [9]. A candidate dissertation on the topic was defended [4]. Several aspects of this research formed the basis for the GPM "Validation of microbiological procedures" in the SP RF XIVth Ed. The GPM contains general approaches to validation/verification of compendial and alternative procedures and also clarifies that "newly developed or revised methods are subject to validation" $[7,10]$. 
The US Pharmacopeia in the section focused on monographs for individual drugs in the monograph Insulin has a test part for enumerating microbes that states the test can be performed on a portion of about $0.2 \mathrm{~g}$, accurately weighed [11].

The goal of the present work was to justify the possibility of reducing the sample size for microbiological analysis of certain PSs.

The following tasks were performed to confirm experimentally the possibility of reducing the sample size for analysis:

- Study the acceptability of using trypticase-soy agar (TSA) growth medium for isolating yeast and mold fungi.

- Investigate the possibility of observing the minimal number of individual microorganism types using TSA agar.

- Design a working scheme for analyzing several categories of PSs based on the obtained experimental results.

\section{EXPERIMENTAL BIOLOGICAL PART}

It had to be proven that yeast and mold fungi could be isolated on TSA as well as on Sabouraud agar to complete the first part of the stated problem. This allowed the experimental confirmation of the reduction in the sample size from $0.4 \mathrm{~g}(\mathrm{~mL})[4]$ to $0.2 \mathrm{~g}(\mathrm{~mL})$ to be shortened.

Test strains of yeast and mold fungi were used for experimental proof of the ability to use TSA to isolate them (Table 1) $[1,10]$.

The detection limits of the microorganisms were determined on the growth media:

-TSA (Merck, Germany);

-Sabouraud agar with dextrose and chloramphenicol (SDCA; Merck, Germany).

The second task was completed by proving that the minimal number (content $\leq 5 \mathrm{CFU}$ per $\mathrm{mL}$ of a 1:10 dilution of the PS) of the pathogenic microorganism species could be isolated from samples of several PSs in Petri dishes with TSA.

TABLE 1. Microorganism Test Strains

\begin{tabular}{lcc}
\hline \multicolumn{1}{c}{ Microorganism } & Strain No. \\
\hline & Mold fungi & \\
Aspergillus brasiliensis & & ATCC 16404 \\
Aspergillus terreus & & VKPM F-1269 \\
& Yeast-like fungi & \\
Candida albicans & & ATCC 10231 \\
Candida utilis & Aerobic bacteria & \\
& & ATCC 8739 \\
Escherichia coli & & ATCC 6538 \\
Staphylococcus aureus & ATCC 9027 \\
Pseudomonas aeruginosa & & \\
\hline
\end{tabular}

The test microorganism Escherichia coli was used for category 2.2 substances; E. coli, Staphylococcus aureus, and Pseudomonas aeruginosa, for category 1.2B compounds (Table 1).

A total of 18 model PSs were studied in the work and included

-category 1.2B: 2-allyloxyethanol, vitamin E acetate, deoxyribonuclease, isotretinoin, insulin Lispro, cabazitaxel, longidase, urea, prostatilen, riboxin, and citicoline sodium;

-category 2.2: atazanavir sulfate, valaciclovir hydrochloride monohydrate, darunavir, inosine pranobex, metoprolol succinate, torasemide, and ezetimibe.

\section{Preparation of inoculate}

1. Cultures (24-d) of microorganisms grown on dense growth medium slants (C. albicans, C. utilis on Sabouraud agar; E. coli, S. aureus, and P. aeruginosa on TSA) were rinsed with sterile normal saline $(0.9 \%)$. Then, microbial suspensions with cell concentration $500 \mathrm{CFU} / \mathrm{mL}$ were prepared using a WHO turbidity standard sample (10 U).

2. Conidia of $A$. brasiliensis ATCC 16404 and A.terreus VKPM F-1269 were obtained by growing cultures on Sabouraud agar for $7 \mathrm{~d}$. Conidia were rinsed with sterile normal saline $(0.9 \%)$ and standardized. Successive dilutions produced working suspensions of the test strains.

The actual contents of cells in the prepared suspensions were found using test inoculations of yeast and mold fungi on SDCA agar.

\section{Inoculation of samples}

1. Inoculate $(0.1 \mathrm{~mL})$ of each microorganism test strain was placed under aseptic conditions into Petri dishes in 20 -fold replicates by two researchers. Each dish was filled with molten agar growth medium $(10 \mathrm{~mL})$ cooled to $45^{\circ} \mathrm{C}$ and incubated for $5 \mathrm{~d}$ in thermostats at $32.5 \pm 2.5^{\circ} \mathrm{C}$ for TSA and $22.5 \pm 2.5^{\circ} \mathrm{C}$ for SDCA agar. Results were observed and recorded daily.

2. Suspensions of microorganism test strains calculated for $0.1 \mathrm{~mL}$ of microorganism suspension containing $500 \mathrm{CFU} / \mathrm{mL}$ per $1 \mathrm{~g}$ of PS were placed into vials containing from $10 \mathrm{~g}(\mathrm{~mL})$ to $20 \mathrm{~g}(\mathrm{~mL})$ of PS (depending on the category). Dilutions (1:10) of the studied PSs were prepared using phosphate buffer as the diluent. Petri dishes $(90 \mathrm{~mm})$ were filled with $1 \mathrm{~mL}$ each (corresponding to a theoretical

TABLE 2. Accuracy and Robustness of Detecting Yeast and Mold Fungi on Trypticase-Soy Agar

\begin{tabular}{lccccc}
\hline \multirow{2}{*}{$\begin{array}{c}\text { Microorganism } \\
\text { test strain }\end{array}$} & \multicolumn{2}{c}{$\begin{array}{c}\text { Accuracy, } \\
\%\end{array}$} & & \multicolumn{2}{c}{$\begin{array}{c}\text { Coefficient } \\
\text { of variation }(\mathrm{CV}), \%\end{array}$} \\
\cline { 2 - 3 } \cline { 5 - 6 } A. brasiliensis & 109 & 102 & & 13.7 & 27.1 \\
A. terreus & 121 & 100 & & 20.6 & 23.5 \\
C. albicans & 110 & 114 & & 21.7 & 16.3 \\
C. utilis & 102 & 108 & & 6.6 & 17.5 \\
\hline
\end{tabular}




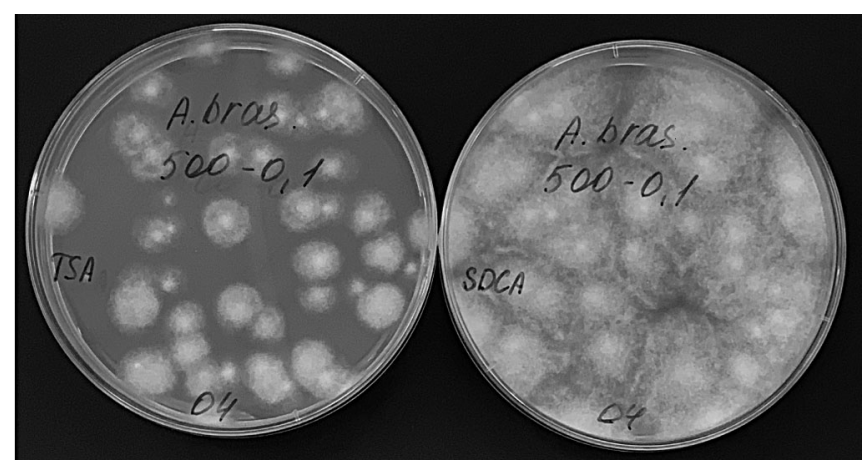

Fig. 1. Visual determination of colonies of A. brasiliensis on trypticase-soy agar (left) and Sabouraud agar (right) after incubation for $5 \mathrm{~d}$.

value of $5 \mathrm{CFU} / \mathrm{mL}$ ) of inoculated solution of PS samples in 10 -fold repetitions by two researchers. The inoculations used a modified deep bowl method with TSA followed by incubation at $32.5 \pm 2.5^{\circ} \mathrm{C}$ for $5 \mathrm{~d}$. Results were observed and recorded daily.

\section{RESULTS AND DISCUSSION}

According to GPM.1.1.0021.18 "Validation of microbiological procedures" [10], improvement of procedures required proof of their applicability, i.e., validation with calculation of critical parameters according to acceptance criteria. For this experiment, the following validation parameters were selected:

-accuracy. This parameter was calculated as the percent ratio of colonies grown on TSA relative to Sabouraud agar taken as the actual (true) number of added microorganism cells. The acceptance criterion was $\leq 70 \%$ of the true value;

-precision (repeatability, convergence). These were evaluated as the degree of scatter of separate results from multiple studies;

-robustness (reliability). These were determined as the degree of intralaboratory precision and reproducibility of analytical results of the same samples under different conditions (different researchers, days, batches of growth media). The acceptance criterion was the coefficient of variation (CV), which should not exceed $35 \%$ [10].

TABLE 3. Precision of Detecting Yeast and Mold Fungi on Trypticase-Soy Agar

\begin{tabular}{lc}
\hline \multicolumn{1}{c}{ Microorganism test strain } & Coefficient of variation $(\mathrm{CV}), \%$ \\
\hline A. brasiliensis & 22.1 \\
A. terreus & 20.6 \\
C. albicans & 16.7 \\
C. utilis & 6.6 \\
\hline
\end{tabular}

The accuracy of isolating yeast and mold fungi was determined for all four species of studied microorganism test strains in 20-fold repetitions by two researchers on TSA and SDCA growth media. The CV was calculated to establish the robustness by varying factors such as different researchers and different batches of growth media (Table 2).

The precision was determined using the $\mathrm{CV}$ for multiple experiments performed in $1 \mathrm{~d}$ using the same materials and equipment and evaluating the degree of convergence of the obtained results (Table 3).

The experimental results showed that all calculated validation parameters satisfied the acceptance criteria. The accuracy was $>70 \%$ of the true value. The $\mathrm{CV}$ was $\leq 35 \%$.

Thus, experimental proof of the ability to isolate yeast and mold fungi using TSA as growth medium was obtained. The ability to reduce the analytical sample size from $0.4 \mathrm{~g}$ $(\mathrm{mL})$ [4] to $0.2 \mathrm{~g}(\mathrm{~mL})$ was proven.

It is also noteworthy that visual counts of colonies in Petri dishes with growth media were more convenient on the $5^{\text {th }}$ day of incubation because they did not coalesce and were more differentiated (Fig. 1).

TABLE 4. Coefficient of Growth of Some Aerobic Bacteria in Tested Pharmaceutical Substances on Trypticase-Soy Agar $(n=10)$

\begin{tabular}{|c|c|c|c|}
\hline \multirow{2}{*}{ Substance } & \multicolumn{3}{|c|}{$\begin{array}{l}\text { Coefficient of growth } \\
\text { of test microorganisms, \% }\end{array}$} \\
\hline & $\begin{array}{l}\text { Escherichia } \\
\text { coli }\end{array}$ & $\begin{array}{c}\text { Staphylococcus } \\
\text { aureus }\end{array}$ & $\begin{array}{c}\text { Pseudomonas } \\
\text { aeruginosa }\end{array}$ \\
\hline 3-Allyloxyethanol & 12 & 10 & 24 \\
\hline Vitamin $\mathrm{E}$ acetate & 94 & 84 & 36 \\
\hline Deoxyribonuclease & 94 & 70 & 68 \\
\hline Isotretinoin & 96 & 62 & 66 \\
\hline Insulin Lispro & 96 & 80 & 46 \\
\hline Cabazitaxel & 28 & 18 & 20 \\
\hline Longidase & 94 & 94 & 64 \\
\hline Urea & 84 & 64 & 26 \\
\hline Prostatilen & 92 & 76 & 106 \\
\hline Riboxin & 94 & 66 & 42 \\
\hline Citicoline sodium & 94 & 60 & 48 \\
\hline Atazanavir sulfate & $*$ & - & - \\
\hline $\begin{array}{l}\text { Valacyclovir hydrochlo- } \\
\text { ride monohydrate }\end{array}$ & 70 & - & - \\
\hline Darunavir & 90 & - & - \\
\hline Inosine pranobex & 76 & - & - \\
\hline Metoprolol succinate & 84 & - & - \\
\hline Torasemide & 78 & - & - \\
\hline Ezetimibe & 80 & - & - \\
\hline
\end{tabular}

${ }_{* *}^{*}$ presence/absence of colonies could not be visually determined; not applicable to category 2.2 PS samples. 
The second stated task was completed by observing and evaluating the colonies of microorganism test strains. The coefficient of growth of the microorganisms was calculated as the percent ratio of the average number of grown colonies with the PS to the test value, which was grown in TSA without the PS.

The accuracy for all studied PSs (qualitative assessment) was $100 \%$ except for PS cabazitaxel (accuracy 70\%). However, the calculated coefficients of growth of all studied microorganisms for PC cabazitaxel and 2-allyloxyethanol were significantly less than the acceptance coefficient (Table 4). The coefficient of growth should be at least $70 \%$ of the added amount of microorganisms [12]. Thus, it could be concluded that these drugs suppressed the growth of a few microorganisms added to the studied sample so that an inactivator had to be selected.

The presence/absence of colonies of E. coli in the Petri dishes could not be visually determined when recording the results for the atazanavir sulfate samples because of floccules of the PS that rose to the surface of the agar.

Table 4 shows that the coefficient of growth of $P$. aeruginosa and $S$. aureus for most studied PSs were less than the established acceptance criteria in 91 and $55 \%$ of the cases, respectively. If the sample contained single colonies of $P$. aeruginosa and $S$. aureus, they could not be isolated on TSA growth medium, which confirmed the need to use the compendial procedure to observe these microorganism strains. For this, the analytical sample mass/volume increased to $1 \mathrm{~g}(\mathrm{~mL})$.

Thus, the minimal allowed amount of a category 2.2 PS was $0.2 \mathrm{~g}(\mathrm{~mL})$; category $1.2 \mathrm{~B}, 1.2 \mathrm{~g}(\mathrm{~mL})$.

A scheme for analyzing several categories of PSs consisting of several steps that enabled the sample size to be reduced was developed based on the experimental results:

1) A validation study, i.e., proof that the analytical sample can be reduced to give results comparable with those from the standard analytical procedure.

2) An algorithm for conducting the analysis with a reduced sample size:

-category 2.2 PS [0.2 g (mL)] or category 1.2B PS [1.2 g $(\mathrm{mL})]$ mixed with an appropriate amount of suitable solvent (p. 2) in a 1:10 dilution.

-inoculation of sample solution $(1 \mathrm{~mL})$ in two Petri dishes by the modified deep bowl method using TSA followed by incubation at $32.5 \pm 2.5^{\circ} \mathrm{C}$ for $5 \mathrm{~d}$.

If colonies appear in the dishes during the $5 \mathrm{~d}$, they must be identified using Gram stain. If Gram-negative bacteria (E. coli suspected) for category 2.2 PS or Gram-negative (E. coli or P. aeruginosa suspected) or Gram-positive bacte- ria (S. aureus suspected) for category 1.2B PS appear, the species must be identified, e.g., on an automated bacteriological analyzer (Vitek 2 Compact, bioMerieux, France) or using manual test systems.

-category 1.2B PS (10 mL, 1:10 dilution) is transferred to $100 \mathrm{~mL}$ of trypsin-soy broth and then analyzed according to recommendations of GPM.1.2.4.0002.18, p. 6.4 and 6.5 "Determination of individual microorganism species."

The results of the validation study led to the following conclusion:

-the permissibility of using TSA to detect yeast and mold fungi and to identify the minimal number of E. coli bacteria was confirmed experimentally;

-the minimal sample size for microbiological examination was determined as $0.2 \mathrm{~g}(\mathrm{~mL})$ for synthetic PS intended for producing nonsterile drugs and $1.2 \mathrm{~g}(\mathrm{~mL})$ for analyzing PS for manufacturing sterile drugs;

-a working scheme was designed for analyzing several PS for which the decrease of analytical sample size was advisable and justified.

\section{ACKNOWLEDGMENTS}

The work was performed in the framework of a State Task for SCEEMP, Ministry of Health of Russia, No. 056-00005-21-00 for applied scientific research (State Acct. No. NIR 121021800098-4).

\section{REFERENCES}

1. SP RF GPM. 1.2.4.0002.18. Microbiological Purity, Moscow (2018), pp. $1128-1200$.

2. 323-FZ, Moscow (2011).

3. MH RF Order No. 69n (2012).

4. I. A. Builova, Candidate Dissertation in Pharmaceutical Sciences, St. Petersburg (2017).

5. O. V. Gunar and I. A. Builova, Khim.-farm. Zh., 50(2), $41-45$ (2016).

6. O. V. Gunar and I. A. Builova, Vopr. Biol., Med. Farm. Khim., 7, $10-15$ (2015).

7. O. V. Gunar, I. A. Builova, L. V. Kolosova, et al., Ved. NTSESMP, 3, 4 - 7 (2013).

8. O. V. Gunar, N. G. Sakhno, et al., RU Pat. 2,596,399, C12Q1 / 04, Sept. 10, 2016.

9. O. V. Gunar, N. G. Sakhno, et al., RU Pat. 2,596,395, C12Q1 / 04, Sept. 10, 2016.

10. SP RF GPM. 1.1.0021.18. Validation of Microbiological Methods, Moscow (2018), pp. $423-443$.

11. USP43-NF38 2S Insulin (2019).

12. European Pharmacopoeia, $10^{\text {th }}$ Ed., 2.6.12. Microbiological examination of nonsterile products (2021). 